\title{
Cellular Inductive Powering System for Weakly-Linked Resonant Rodent Implants
}

\author{
Nima Soltani, Miaad S. Aliroteh, and Roman Genov \\ Department of Electrical and Computer Engineering \\ University of Toronto, Toronto, Ontario M5S 3G4 Canada \\ Email: \{nima,roman\}@eecg.utoronto.ca, miaad.seyedaliroteh@utoronto.ca
}

\begin{abstract}
This paper presents a cellular inductive powering system for neural interface devices to facilitate chronic physiological studies. The system delivers $21-225 \mathrm{~mW}$ of power to a $4 \mathrm{~cm} \times 4 \mathrm{~cm}$ planar receiver with $21.5 \%$ efficiency. It is shown that the implemented multi-coil power transmission technique creates 5 times less non-ionizing radiation at $10 \mathrm{~cm}$ distance than a single-coil design, for equal amounts of delivered power. The design also implements a low-cost technique which tracks the location of the animal using an impedance measurement circuit which is also used to tune the individual coils.
\end{abstract}

\section{INTRODUCTION}

Chronic studies using neural interfaces (NIs) on awake behaving rodents have become a common technique. In recent years, research efforts have made significant advances in this area and it continues to progress [6], [7], [8].

Experiments on laboratory rodents are often conducted with a cable carrying power to the NI device. Cables have the typical disadvantage of the animal gnawing and pulling on the them thereby disrupting the experiment. Also the risk of an infection is always present [4]. Alternatively, on-board battery can be used to power the NI implant which inevitably limits the duration of the experiment. Typically, battery life limits the length of studies to 7 hours.[3]. Therefore, longterm studies need to be interrupted to conduct maintenance sessions. Longer-lasting batteries are heavier and are not used in studies with smaller rodents.

This paper presents a wireless power delivery system shown in Fig. 1 for neural interfaces implanted in laboratory rodents. The design eliminates the above-mentioned problems, and facilitates long-term physiological studies. The proposed system supplies more power and offers greater hardware scalability by using fewer components as compared with previously reported designs [1], [2]. Such an inductive powering solution has a potential to become a requirement for all live rodent studies.

\section{THEORY}

\section{A. Mutual Inductive Coupling}

Two inductors are mutually coupled when they are in the reactive near-field of one another, as shown in Fig. 2. A portion of the magnetic field created by the first inductor passes through the cross-section of the second inductor, inducing voltage in the far inductor and enabling inductive power transfer.

The first author accepts the full responsibility for technical and literary editing of this manuscript

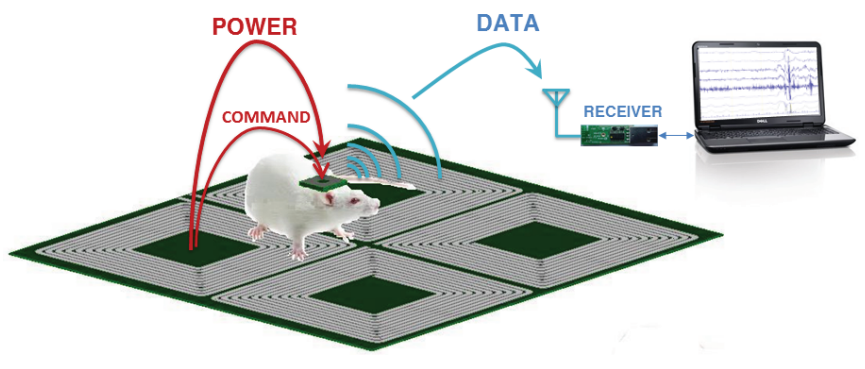

Fig. 1. Conceptual illustration of the proposed inductive powering system which consists of an array of power transmitting planar coils located on the bottom of the cage. Animal is dynamically tracked and only the nearest coil is turned on. Neural recording data are transmitted to a nearby computer while simple commands are communicated over the same inductive link used to power the device.

\section{B. Resonant Inductive Coupling}

According to Faraday's law, in the mutually coupled system of Fig. 2, the voltage induced into the far inductor, $V_{21}$, is directly proportional to the operating frequency. At high frequencies, however, the self-reactances of the two coils, $\omega L_{1}$ and $\omega L_{2}$, are so large that very little current can flow in either coil, allowing only trace amounts of power to be transferred. To overcome the problem of large self-reactance, capacitors are used on both sides to make the coils resonant as shown in Fig. 3(a).

At resonance, the reactances of the transmitter and the receiver are nullified as shown in Figs. 3(b) and (c), leaving only the resistive divider of Fig. 3(d). In this divider network, the load component, $R_{e q 2}^{\prime}$, becomes smaller as the magnetic coupling coefficient decreases making the load increasingly hard to match and limiting the overall power efficiency. In general [5]:

$$
R_{e q 2}^{\prime}=\frac{\omega^{2} L_{1} L_{2}}{R_{e q 2}} k^{2} .
$$

Rearranging (1) and assuming the conjugate matched condition on both sides, one can write:

$$
Q_{1} Q_{2}=\frac{1}{k^{2}}
$$




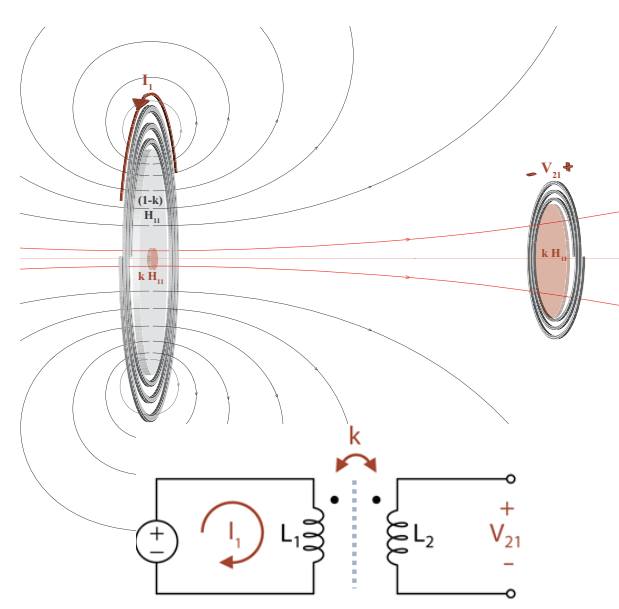

Fig. 2. Power transfer using the principle of mutual inductive coupling.

where $Q_{1}=\frac{\omega L_{2}}{R_{e q 2}^{\prime}}$ and $Q_{2}=\frac{\omega L_{2}}{R_{e q 2}}$ are quality factors of the primary and the secondary networks under conjugate-matched condition.

Fig. 4 shows Thevenin equivalent of the system as seen at the secondary side. According to this model, the parasitic resistance of $L_{2}$ is in series with the Thevenin equivalent resistance, $R_{21}$, causing the power loss of the secondary coil to be equal to $\frac{R_{s 2}}{R_{21}+R_{s 2}}$. For high power transmission efficiency, therefore, the component quality factor of the secondary coil, defined as $Q_{L 2}=\frac{\omega L_{2}}{R_{s 2}}$, must be much greater than the circuit quality factor, $Q_{2}$. A similar condition can be established for the primary coil. Combining these conditions with (2), we arrive at the coil design condition for high-efficiency power transfer:

$$
Q_{L 1} Q_{L 2} \gg \frac{1}{k^{2}}
$$

where $Q_{L 1}$ and $Q_{L 2}$ are the component quality factors of the coils. Due to the relatively large separation of the two coils in the targeted experiments, a typical coupling factor, $k$, in the range of 0.005 to 0.1 is expected, which according to (3) requires the design to have high-Q coils.

Another challenge in wireless power transfer is keeping the coils resonant at all times. Presence of conductive objects or media with high dielectric constant changes the value of parasitic capacitances, $C_{p 1}$ and $C_{p 2}$, causing the resonant frequency of the coils to shift away from the operating frequency, as shown in Fig. 5. Dynamic resonance tuning is used to keep the coils resonant at all time. This will be discussed in Section IV.

\section{COILs Design}

According to (3), the required quality factor of the transmit (TX) and receive (RX) coils is determined by the minimum value of the coupling coefficient, $k$, which occurs at the maximum operating distance. Two possible implementations of powering systems are shown in Figs. 6(a) and (b), including the proposed design in Figs. 6 (b). Based on the magnetic field simulation results in Figs. 6(c) and (d), for a coil separation of

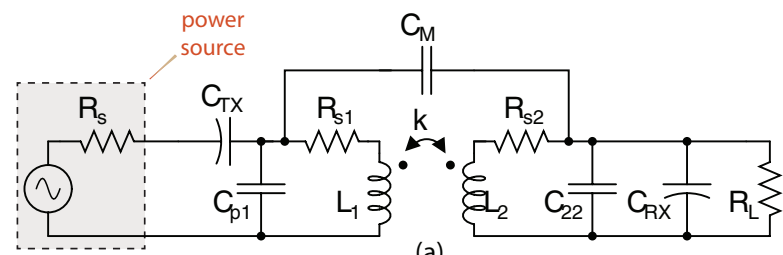

(a)

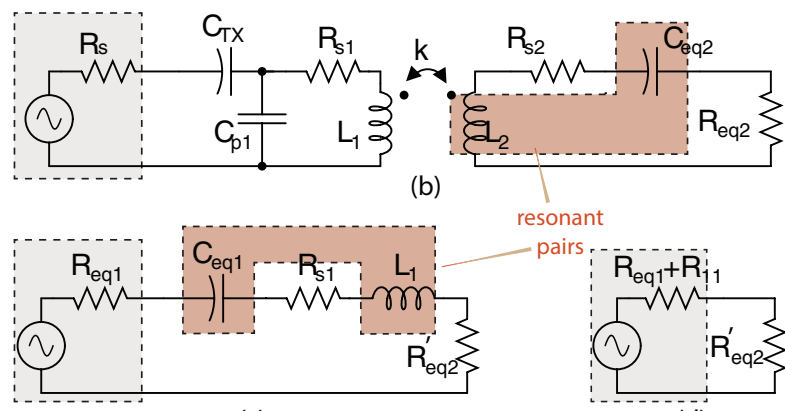

(c)

(d)

Fig. 3. (a) Lumped model of the inductive powering system as a weaklylinked transformer $(k \ll 1)$ with parasitic components. (b) Same model with $C_{p 2}, C_{R X}$ and $R_{L}$ transformed into a series network of $C_{e q 2}$ and $R_{e q 2}$ where $C_{e q 2}$ is in resonance with $L_{2}$ which only leaves $R_{s 2}$ and $R_{e q 2}$ left on the RX side. (c) The transformed model in (b) further simplified by moving the resistive load of RX to the TX side $\left(R_{e q 2}^{\prime}\right)$. and transforming $R_{s}, C_{T X}$ and $C_{p 1}$ into a series network of $R_{e q 1}$ and $L_{1}$ where $C_{e q 1}$ is in resonance with $L_{1}$. (d) Effective model of the inductive powering system as resistive divider where one resistance $\left(R_{e q 2}^{\prime}\right)$ represents the load on the RX side and the other represents the source impedance on the TX side. As $k$ decreases, this network become more difficult to match.

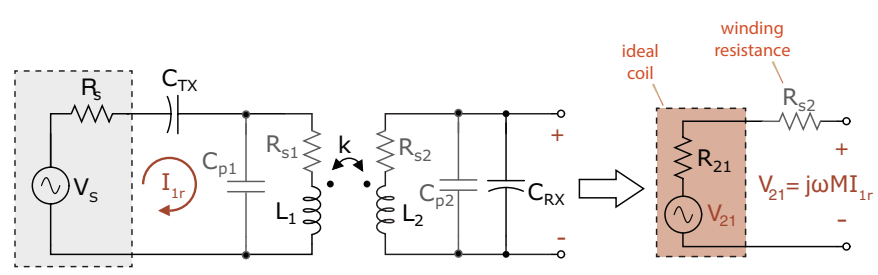

Fig. 4. The ${ }^{\prime}$ venin equivalent circuit of the secondary coil. Parasitic resistance $R_{s 2}$ is in series with the Thevenin equivalent resistance (of ideal coil), making the fraction of lost power equal to the ratio of parasitic to overall resistances.

$10 \mathrm{~cm}$, an approximate coupling coefficient of 0.1 is expected. As the results in Figs. 6(c) and (d) refer only to a receiver with outer diameter of $40 \mathrm{~mm}$, the $k$ values must be scaled to the particular RX outer diameter of interest. The TX outer diameter, on the other hand, does not have a significant impact on $k$ as long as it is larger than the coil separation. In this design, we select TX outer dimension to be approximately equal to the maximum expected separation of the coils, which is $100 \mathrm{~mm}$. As shown in the 3D field simulations in Figs. 6(e) and (f), a TX coil larger than this size will create excessive and unnecessary field emissions. TX coil dimensions smaller than this value will result in impractically small $k$, for which we will not be able to satisfy the condition in (3).

Fig. 7 shows inductance and quality factors of planar rectangular coils with different number of turns and outer diameters for both TX (Figs. 7(a) and (b)) and RX (Figs. 7(c) and (d)) coils. From Figs. 7(c) and (d), the quality factor is concluded to be almost insensitive to the increased number of 


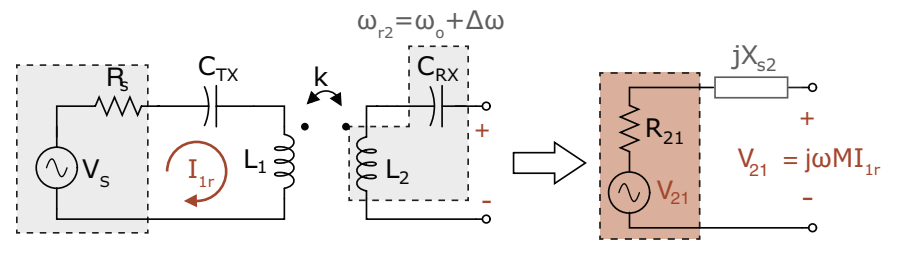

Fig. 5. Parasitic capacitances $C_{p 1}$ and $C_{p 2}$ are affected by nearby conductive objects and dielectric media, changing the resonant frequency and reducing output power

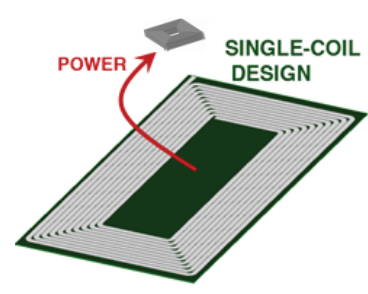

(a)

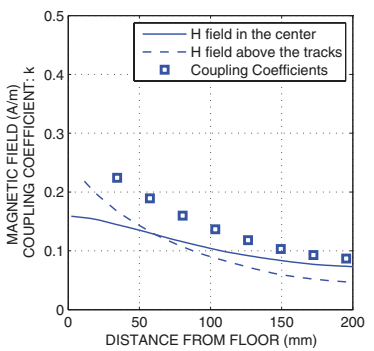

(c)

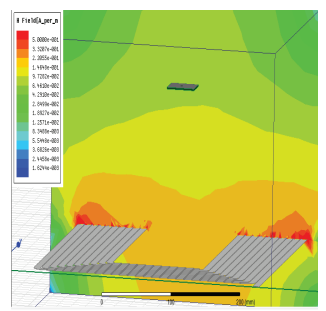

(e)

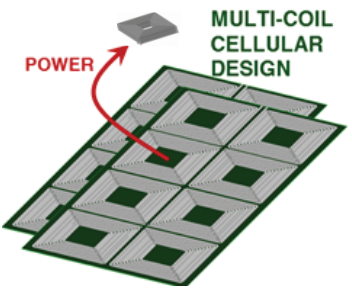

(b)

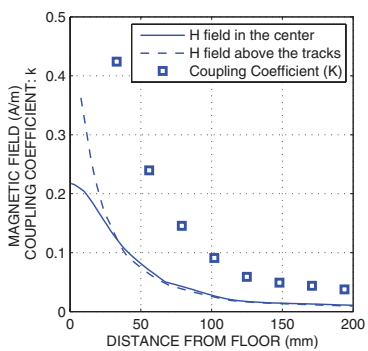

(d)

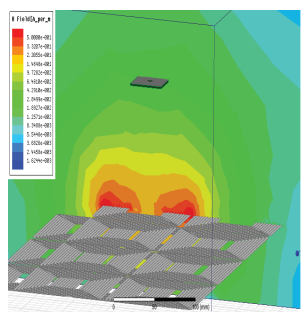

(f)
Fig. 6. (a) Simple single-TX inductive power system. (b) The implemented multi-coil technique. The multi-coil floor creates less non-ionizing radiation than the single-coil design. This is validated by magnetic field $(H)$ and inductive coupling coefficient $(k)$ for the tile-based (c) and the single-coil (d) approach. (e) and (f): the 3D field simulation results for each method.

turns, and is only improved by increasing the outer diameter. This is particularly significant for the receiver which needs to be as small as possible. Using (3), and realizing that the Qfactor requirement for either coil is most relaxed when $Q_{L 1}$ and $Q_{L 2}$ are equal, we select the smallest receiver coil that satisfies $Q_{L 2}>1 / k$, which corresponds to the outer diameter of approximately $40 \mathrm{~mm}$.

\section{Multi-Coil System Design}

Since the area to be powered is much greater than the size of the TX coil, as desecribed in Section III, a multi-coil powering technique, as shown in Fig. 6(b), is proposed. Two overlapping arrays of $10 \mathrm{~cm} \times 10 \mathrm{~cm}$ TX coils are used to power an overall floor area of $45 \mathrm{~cm} \times 26 \mathrm{~cm}$. At any given time, only the coil
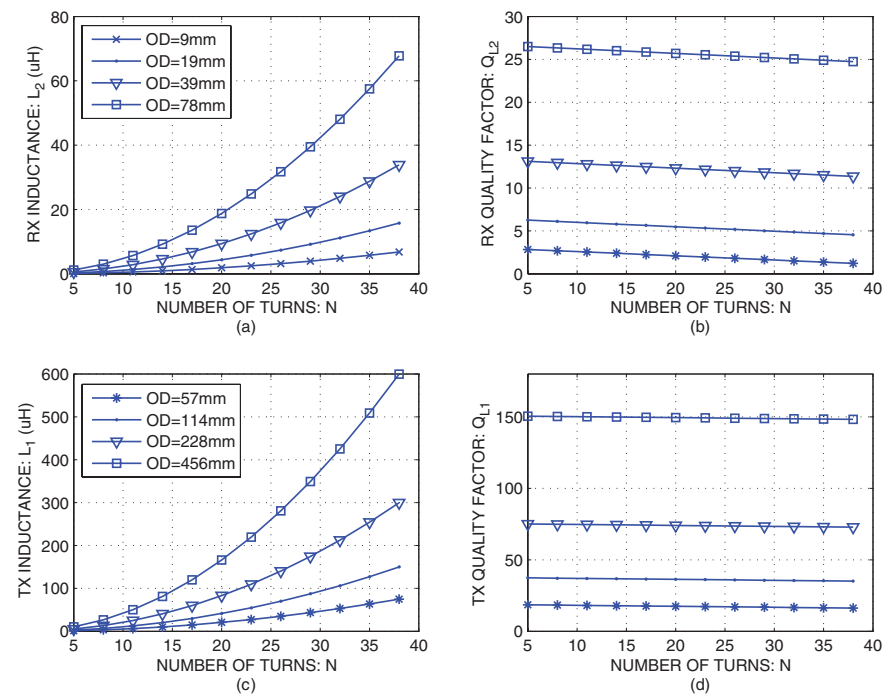

Fig. 7. Inductance (a and c) and quality factors (b and d) of transmit and receiver coils at $1.5 \mathrm{MHz}$ for different outer diameters.

nearest to the animal is turned on.

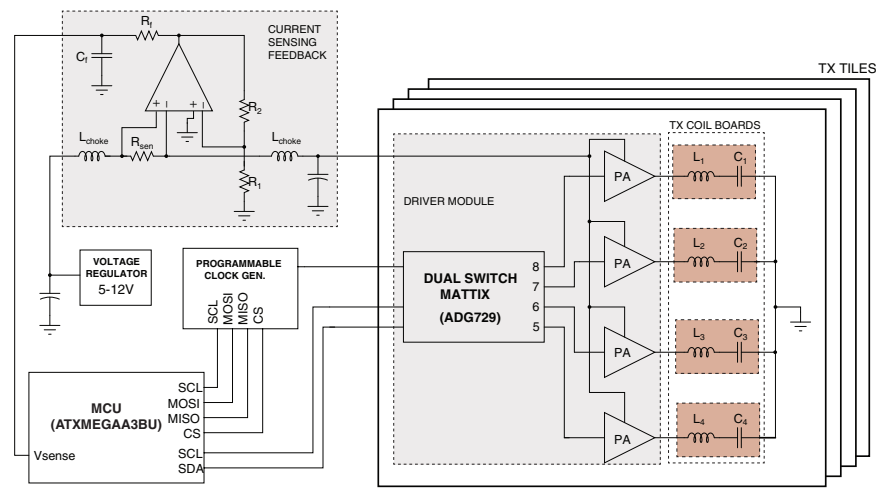

Fig. 8. Schematic block diagram of the cellular inductive powering system, made up of 4 tiles and a control unit. Each tile consists of 4 TX coils, each connected to a power amplifier. A switch matrix turns on one of the 4 coils when the tile is active. The MCU uses a current sensing feedback signal from the power supply to dynamically tune the coils, and to determine which coil must be activated.

Fig. 8 shows the functional block diagram of the cellular inductive powering system. To make the system more scalable, TX coils are organized into "tiles". Each tile consists of four coils, and connects to a driver module made up of 4 power amplifiers (PA) and a matrix switch. PAs are designed with $\mathrm{H}-$ bridge drivers (L6741) and discrete power FETs (DMN4031). A central MCU activates a particular PA via an I2C bus routed to each driver module. The driver module then selects the appropriate PA via the dual matrix switch (ADG729) by routing a $1.5 \mathrm{MHz}$ squarewave to that $\mathrm{PA}$.

The MCU works in one of the following 3 states: (1) calibration which runs at power-up, whereby the MCU determines the exact resonant frequency and impedance of each coil, (2) a local search for receiver by measuring the current in each coil near the last known position of the receiver using the "current sensing feedback" in Fig. 8, (3) a global search for the coil 
TABLE I

PERFORMANCE SUMMARY AND COMPARISON

\begin{tabular}{lccc}
\hline Reference & {$[1]$} & {$[2]$} & This work \\
& & & \\
\hline Operating frequency: $f_{o}(\mathrm{MHz})$ & 13.65 & $0.1-0.2$ & 1.55 \\
Field exposure limit at $f_{o}(\mathrm{~A} / \mathrm{m}) *$ & 1.2 & 80 & 10.9 \\
RX-TX separation $(\mathrm{mm})$ & 70 & 50 & 73 \\
Array Dimensions & - & $3 \times 3$ & $2 \times 8 \times 2$ \\
Coupling Coefficient:k & 0.21 & - & 0.17 \\
TX diameter $(\mathrm{mm})$ & 168 & 100 & 115 \\
RX diameter $(\mathrm{mm})$ & 40 & - & 40 \\
Load resistance: $R_{L}(\mathrm{~K} \Omega)$ & 0.5 & 1 & 3.3 \\
Transferred Power:P(mW) & $20-145$ & 100 & $21.6-225$ \\
Coil-to-Coil efficiency: $\eta_{c o i l}$ & $17.8-42.5$ & - & $7.2-29.2$ \\
Overall efficiency: $\eta_{s y s t e m}$ & - & - & $5.3-21.5$ \\
TX inductance: $L_{11}(\mu \mathrm{H})$ & 1.28 & 27 & 10.62 \\
Quality factor: $Q_{11}$ & 168 & - & 129 \\
Resonating capacitance: $C_{T X}(\mathrm{nF})$ & 0.108 & $22-122$ & 1.05 \\
RX inductance: $L_{22}(\mu \mathrm{H})$ & 1.12 & - & 10.35 \\
Quality factor: $Q_{22}$ & 140 & - & 77 \\
Resonating capacitance: $C_{R X}(\mathrm{pF})$ & 123 & - & 1043 \\
\hline
\end{tabular}

with the highest impedance which only runs when the local search is not successful.

\section{RESUlTs}

The magnetic field plots in Figs. 6(e) and (f) demonstrate that the smaller outer diameter of the transmitting coils in Fig. 6(f) results in significantly less non-ionizing radiation than the single-coil approach in Fig. 6(e), making the implemented technique safer for the clinician who conducts the experiments, as well as the animal. Figs. 6(c) and (d) illustrate the critical advantage of the multi-coil technique quantitatively. It can be verified that, in Fig. 6(d), the ratio of coupling coefficient to the magnetic field intensity is approximately 3 times larger than that in Fig. 6(c). This effectively validates the initial motivation behind the more complex multi-coil technique.

Measured characteristics of designed system and similar recent work are listed in Table I. Operating at lower frequency, the design exhibits less coil-to-coil efficiency than that in [1]. However, since the non-ionizing field exposure limit is much higher at lower $\mathrm{MHz}$ frequencies, the system can in fact transmit more power by safely creating more magnetic field at the receiver.

TABLE II

DESIGN SCALABILITY

\begin{tabular}{lcc}
\hline Reference & {$[1]$} & This work \\
\hline Location-sensing & 3D Magnetic Sensors & $\begin{array}{c}\text { Reflected impedance } \\
\text { method }\end{array}$ \\
$\begin{array}{l}\text { Power control } \\
\text { mechnism }\end{array}$ & $\begin{array}{c}\text { RFID Readers } \\
(\times \mathrm{N})\end{array}$ & $\begin{array}{c}\text { Reflected impedance } \\
\text { (software implementation) }\end{array}$ \\
\# of Sig. Gen. & $\mathrm{N}$ & 1 \\
\# of MPU's & $\mathrm{N} / 3$ & 1 \\
Coil material & 4-layer PCB & 2-layer PCB \\
*Number of TX coils. & &
\end{tabular}

Table II shows that by eliminating the need for multiple
MCUs, RFID readers, and magnetic sensors, the proposed system offers greater scalability at a lower cost. The current sensing feedback simplifies the task of dynamic tuning and load-tracking at a fraction of the cost. In another comparison, the design in [2] operates at an even lower frequency which further reduces field emissions. However, that assigns a different operating frequency to each transmitting coil which limits the number of coils to the number of distinct resonant frequencies that can be created without causing interference between coils. Also, expanding the range of resonance frequencies beyond that of the first value will require a different coil design for higher frequencies, thereby limiting scalability.

\section{CONCLUSIONS}

A practical wireless power transfer system was designed and implemented for safe delivery of wireless power to implantable neural interface microsystems. The proposed design is shown to inductively transfer sufficiently large amounts of power while effectively minimizing the levels of non-ionizing radiation in the vicinity of the transmitting coils.

\section{REFERENCES}

[1] U. Jow, M. Kiani, X. Huo, and M. Ghovanloo, "Towards a smart experimental arena for long-term electrophysiology experiments," Biomedical Circuits and Systems Conf., pp. 121- 124, 2011.

[2] D. McCormick, A. Hu, P. Nielsen, S. Malpas, and D. Budgett, "Powering implantable telemetry devices from localized magnetic fields," Ann. Int. Conf. Engineering in Medicine and Biology Society, pp. 2331- 2335 , 2007.

[3] D. Budgett, A. Hu, P. Si, W. Pallas, M. Donnelly, J. Broad, C. Barrett, S. Guild, and S. Malpas, "Novel technology for the provision of power to implantable physiological devices," J. Applied Physiology, vol. 102, no. 4, p. 1658, 2007.

[4] A. Schwartz, X. Cui, D. Weber, and D. Moran, "Braincontrolled interfaces: movement restoration with neural prosthetics," IEEE/ICCME Int. Conf. Complex Medical Engineering (CME), pp. 79-84, 2010.

[5] N. Soltani, and F. Yuan, "A High-Gain Power-Matching Technique for Efficient Radio-Frequency Power Harvest of Passive Wireless Microsystems," IEEE Tran. Circuits and Systems-I: Regular Papers, vol. 57, no. 10, pp. 2685- 2695.

[6] K. Abdelhalim, V. Smolyakov and R. Genov, "A phase synchronization and magnitude processor VLSI architecture for adaptive neural stimulation," IEEE Biomedical Circuits and Systems Conf., pp. 5-8 , 2010.

[7] K. Abdelhalim, L. Kokarovtseva, J. Perez Velazquez, R. Genov, "915-MHz FSK/OOK Wireless Neural Recording SoC With 64 Mixed-Signal FIR Filters," IEEE J. Solid-State Circuits, no. 99, pp. 1-16, 2013.

[8] C. Qian, J. Parramon, E. Sanchez-Sinencio, "A Micropower Low-Noise Neural Recording Front-End Circuit for Epileptic Seizure Detection," IEEE J. Solid-State Circuits, vol. 46, no. 6, pp. 1392 - 1405, 2011. 